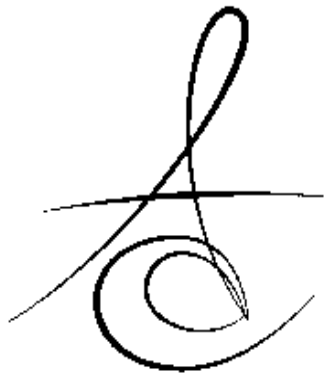

\section{GÜNCEL NANOSERAMİK VE HİBRİT CAD/CAM MATERYALLERİ}

CURRENT NANOCERAMIC AND HYBRID CAD/CAM MATERIALS

\author{
Arş. Gör. Dt. Hamiyet KILINÇ ${ }^{*}$
}

Dr. Öğr. Gör. Elif AYDOĞAN AYAZ*
Doç. Dr. Sedanur TURGUT*

Prof. Dr. Bora BAĞIŞ**

Makale Kodu/Article code: 2871

Makale Gönderilme tarihi: 31.05.2016

Kabul Tarihi: 28.07.2016

öz

CAD/CAM teknolojisi; indirekt restorasyonların üretim sürecini kolaylaştırması, daha estetik ve üniform kalitede restorasyonların elde edilmesi gibi avantajlarla, diş hekimliği alanında her geçen gün daha da popüler hale gelmektedir. Nanoteknolojideki ilerlemeler ile üretilen gelişmiş özelliklere sahip nanoseramik ve hibrit malzemelerin kullanımı, seramik ve kompozit materyallerinin avantajlarını bir araya getirmiştir. Bu derlemede; CAD/ CAM ile kullanılabilen farklı içerik ve ticari markadaki güncel nanoseramik ve hibrit materyallerin, genel özellikleri ve klinik prosedürleri hakkında bilgi vermek amaçlanmıştır.

Anahtar Kelimeler: CAD/CAM; nanoseramik; hibrit materyali

\section{GİRİŞ}

Diş hekimliğinin en önemli amaçlarından biri; kaybedilmiş doku bütünlüğünün olabildiğince kısa sürede yeniden sağlanmasıdır. CAD/CAM teknolojisi; indirekt restorasyonların üretim sürecini kolaylaştırması ve üniform kalitede estetik restorasyonların elde edilmesi gibi avantajlarla, her geçen gün daha da popüler hale gelmektedir. ${ }^{1,2}$ CAD-CAM sistemleri ile yeni malzemelerin kullanımına olanak sağlanmaktadır.,4 Teknolojideki gelişmeler doğrultusunda, protetik tedavilerde kullanılacak yeni materyaller geliştirilmektedir. ${ }^{5,6}$

CAD/CAM sistemlerinde farklı yapılarda çeşitli materyaller kullanılabilir. ${ }^{7,8} \mathrm{Bu}$ materyaller şu şekilde sınıflandırılabilir:

\section{ABSTRACT}

CAD/CAM technology is becoming more popular with the advantages of facilitating the production process of indirect restorations, obtaining more esthetic and uniform restorations, in the field of dentistry each passing day. With developments in nanotechnology, produced ebhanced features nenoceramic and hybrid materials has brought the advantages of ceramic and composite materials together. This review purposed to give information about the general characteristics and clinical procedures of current nanoceramic and hybrid materials with different content and trademarks.

Keywords: CAD/CAM; nanoceramic; hybrid materials
1. Feldspatik seramikler

2. Cam matriksi güçlendirilen seramikler

a. Lösit ile güçlendirilmiş cam seramikler

b. Lityum disilikat ile güçlendirilmiş cam seramikler seramikler

3. İnfiltrasyon seramikleri

4. Oksit seramikler

a. Alimünyum oksit $\left(\mathrm{Al}_{2} \mathrm{O}_{3}\right)$

b. Zirkonyum oksit $\left(\mathrm{ZrO}_{2}\right)$

5. Nanoseramikler

6. Hibrit seramikler

7. Zirkonya ile güçlendirilmiş lityum silikat seramikler

8. Kompozitler

9. Polimerler

10. Metaller

Kendine özgü avantajları ile estetik diş hekimliğinde en yaygın kullanım alanına sahip materyaller;

\footnotetext{
* Karadeniz Teknik Üniversitesi, Diş Hekimliği Fakültesi, Protetik Diş Tedavisi AD, Trabzon

**̇̇mir Katip Çelebi Üniversitesi, Diş Hekimliği Fakültesi, Protetik Diş Tedavisi AD, İzmir
} 
seramik ve kompozitlerdir. Her iki materyalinin de avantalarını içeren, doğal dişin fiziksel ve yapısal özelliklerini taklit eden; nanoseramikler ve 'polimer-infiltreseramik-ağ' yapısına sahip hibrit seramikler geliştirilmiştir. ${ }^{9}$ Bu malzemelerin mekanik özelliklerinin, doğal diş dokusu ile benzer değerler gösterdiği ve üst yapı materyalinin tabakalar halinde atma ve kırılma riski daha düşük olduğu bildirilmiştir. ${ }^{10}$ Tesviye ve polisaj işlemleri daha kolaydır. Fırınlama gerektirmez. Özellikle tek seansta, klinikte restorasyonun üretilmesine dayanan uygulamalar için uygundur. ${ }^{11}$

\section{NANOSERAMİKLER}

Nanoteknolojinin temeli, atomları ve molekülleri tek tek kontrol ederek fonksiyonel yapılar oluşturma ve büyük ölçekli yapılardan daha farklı fiziksel, kimyasal ve biyolojik özellikler gösteren materyallerin, cihazların ve sistemlerin geliştirilmesi fikrine dayanır. Biyoteknoloji ve nanomateryallerin kullanımı ile nanodişhekimliği neredeyse mükemmel bir ağız sağlığının mümkün olmasını sağlayacaktır. ${ }^{12,13}$ Diş hekimliğinde nanoteknoloji ilk olarak 1997 yılında materyallerin fiziksel özelliklerini geliştirmek amacıyla kullanılmaya başlanmıştır ve bu teknoloji ile yeni restoratif materyaller geliştirilmiştir. ${ }^{14}$ Nanoseramik materyallerin elastiklik modülü 10 $20 \mathrm{GPa}$ dır ve dentine yakın değerler göstermektedir. $\mathrm{Bu}$ materyallerin cam seramiklere oranla stresi daha fazla absorbe ettiği belirtilmiştir. Kırılma direnci 204 MPa olup, bu değer; feldspatik, lösit ve kompozit içerikli bloklardan yüksek, lityum disilikatla güçlendirilmiş bloklara ise yakındır. Ayrıca karşıt dişte meydana getirdikleri aşınma miktarının, cam seramiklere kıyasla çok daha az olduğu bildirilmiştir. ${ }^{15,16}$

LAVA Ultimate (3M ESPE, Neuss, Minn, A.B.D); nanoseramik teknolojisi ile üretilen yeni bir materyaldir ve rezin nanoseramik (RNC) olarak adlandırılır. Malzeme bir reçine ya da kompozit olmadığı gibi saf seramik de değildir. Her ikisinin bir karışımıdır ve ağırlıklı olarak seramik içerir (\%80 seramik, \%20 kompozit). Seramik yapı, zirkonya ve silika nanomerlerden oluşmaktadır. Kompozitin esneklik ve yüksek kırılma dayanımı özelliklerini, seramiğin estetik özelliklerini birleştirmek amacı ile üretilmiştir. Üretim sonrası fırınlama gerektirmez ve kolaylıkla parlatılabilir. LAVA Ultimate restoratif materyali; tek seansta muayenehanede uygulanabilen bloklar (Ör. CEREC® ve E4D) ve laboratuarda kullanılan bloklar (Ör. Straumann ( CARES $R$ ) bloklar olmak üzere farklı şekillerde üretilmişlerdir. ${ }^{17}$
İnley, onley ve veneer restorasyonların yapımında kullanılabilir. Tam kron yapımı, dişe olan zayıf bağlantısı nedeniyle kontrendikedir. Yüzey bitim protokolü diğer CAD/CAM materyallerine kıyasla daha hızlıdır. Restorasyon yapım aşamasında fırınlama gerektirmez. Freze, parlatma ve uyumlama işlemleri kolaydır. Işıkla sertleşen restoratif materyaller ile ağız içi uyumlamalar, ilaveler ve tamir yapılabilmesi sistemin en önemli avantajlarındandır. ${ }^{18,19}$

RNC bloklar, Vitapan skalasına göre düşük traslüsensi (LT) A1, A2, A3, A3.5, B1, C2, D2, Bleach ve yüksek translüsensi $(H T) A 1, A 2, A 3, B 1$ olmak üzere iki ayrı translüsensi ve sekiz ayrı renk özelliğinde üretilmiştir. Yüzey bitim işlemleri için lastik frezler ve cila pastası kullanılabilir. Üretici firma tarafından, kişisel karakterizasyon için gerekli glazür malzemelerinin, restorasyonun pit ve fissürlerine kolaylıkla uygulanabileceği belirtilmiştir. Ancak, servikal bölgede boyama önerilmemektedir. Simantasyon aşamasında restorasyona, $50 \mu \mathrm{m} \mathrm{Al}_{2} \mathrm{O}_{3}$ ile 2 bar basınç altında kumlama yapılır. Adeziv simanlar ile simante edilmesi önerilmektedir. ${ }^{19}$

Cerasmart (GC Dental Poducts, A.B.D.); esnek nanoseramik olarak literatürde yer almaktadır. Kuvvet absorbsiyonu ve esneklik özellikleri ile ön plana çıkmaktadır. Esnek nanoseramik, matriks yapısı sayesinde gelen kuvvetleri homojen ve eşit olarak dağıtır. Seramik ve kompozit materyalinin pozitif özelliklerini birleştirmek amacıyla üretilmiştir. Yüksek esneklik özelliği marjinal adaptasyonun iyi olmasına ve simantasyon sonrası yüksek mukavemet gösterebilmesine olanak sağlar. İçeriğinde \%71 oranında silika, \%29 oranında kompozit bulunur. ${ }^{20}$

İnley, onley, veneer restorasyonlar, kron restorasyonları, implant üstü restorasyonların yapımında kullanılabilir. Yüksek esneklik ve kırılma dayanımı, yüksek radyoopasite, yüksek aşınma direnci, üst yapı materyalinin tabakalar halinde atma riskinin düşük olması, sinterleme ve fırınlama işlemlerine gerek olmaması, intraoral tamir yapılabilmesi sistemin avantajlarıdır. ${ }^{20}$

Derin chamfer ya da yuvarlatılmış shoulder tipi basamak preperasyonu ile daha iyi sonuçlar elde edileceği belirtilmiştir. İnley veya onley restorasyonlarda, diş ile restorasyonun birleşim yerlerinin, karşıt diş ile direk oklüzal kontakta olmamasına dikkat edilmelidir. Yüksek translüsensi $(H T) A 1, A 2, A 3, A 3.5, B 1$ ve düşük translüsensi (LT) $A 1, A 2, A 3, A 3,5, B 1$ özelliğinde bloklar mevcuttur. Yüksek translüsensi özelliğine 
sahip bloklar genellikle, restorasyon sadece mine dokusun yerini alacaksa ve dişte maskeleme gerektirecek herhangi bir renklenme yoksa, düşük translüsensi özelliğine sahip bloklar ise, kesim, dentini de içeriyorsa ve ilgili dişlerde renklenme mevcutsa tercih edilir. Cerasmart materyali ile restorasyon üretildikten sonra, renk karakterizasyonu ve glaze işlemleri için, sistemin içinde yer alan 'Optiglaze color' kullanılabilir. Bu aşamada öncelikle restorasyon; 25- $50 \mu \mathrm{m} \mathrm{Al}_{2} \mathrm{O}_{3}$ ile kumlanır. Ardından ultrasonik banyo içinde temizlenir. Son olarak çeşitli renk seçenekleri bulunan 'Optiglaze color' ile estetik düzenlemeler, istenen şekilde tamamlanır. Uygulama sonrası bu ajanlar ışı cihazı ile sertleştirilirler. ${ }^{20,21}$

Üretici firma tarafından simantasyon aşamasında 25-50 $\mu \mathrm{m} \mathrm{Al}_{2} \mathrm{O}_{3}$ ile kumlama ya da \%5 hidroflorik asit ile pürüzlendirme yapılabileceği belirtilmiştir. Yüzey uygulamaları ardından adeziv simantasyon prosedürü talimatlara göre uygulanıp simante edilebilirler. $^{20}$

\section{HİBRİT SERAMİKLER}

$\mathrm{Bu}$ blokların yapısında baskın oranda bulunan seramik ağı, birbiri içerisine tamamen entegre olan bir polimer ağı ile güçlendirilmiştir. Seramik materyalinde sık karşılaşılan çatlak ilerlemesi sorunu polimer ağ yapısı sayesinde azaltılmıştır. ${ }^{21}$ Diğer materyallerde olduğu gibi, hibrit seramiklerin de optik özellikleri; monomer kompozisyonu, kimyasal içerik, doldurucuların partikül büyüklüğü ve dağıımından etkilenmektedir. ${ }^{22}$

Vita Enamic (Vita Zahnfabrik, Bad Sackingen, Germany); hibrit yapısı pöröz bir seramik altyapı üzerine infiltre edilen monomer yapının ışıkla sertleştirilmesi ile oluşturulmuştur. ${ }^{23}$ Vita Enamic, dentine benzer elastisite modülüne sahiptir. Ağırlıkça $\% 86$, hacimce $\% 75$ oranında inorganik içerik yani seramik yapıdan, ağırıkça \%14, hacimce \%25 oranında organik içerik yani polimer yapıdan oluşur. Seramik kısım ağırlıkça; \% 58-63 silikon dioksit, \%20-23 alüminyum oksit, \%9-11 sodyum oksit, \% 4-6 potasyum oksit, \% 0.5-2 baron trioksit, <\%1 zirkonya ve <\%1 kalsiyum oksitten oluşur. ${ }^{24}$ Organik kısım ise UDMA (Üretan dimetakrilat) ve TEGDMA (Trietilen glikol dimetakrilat) materyallerinden oluşur. ${ }^{25}$ Kırılma dayanımı tam seramiklere göre daha yüksektir ve CAD/CAM sistemleri ile hazırlanma prosedürü daha kolaydır. Vita Enamic sadece tek diş restorasyonlarında kullanılabilir. ${ }^{26,27}$

Restorasyon üretildikten sonra Vita Enamic
Stains likiti ile staining tekniği kullanılarak renk karakterizasyonu yapılabilir. Materyale fırınlama yapılmaz. Işıkla polimerize olan staining ve glazür ajanları mevcuttur. Gerekirse 'Vita VM LC' veneer kompoziti ile ilaveler yapılabilir. Simantasyon aşamasında; diş fosforik asit ile, restorasyon hidroflorik asit ile (60 sn) asitlenir. Adeziv simanlar ile simantasyon uygulanması önerilmektedir. ${ }^{27,28}$

Diş hekimliğine yeni tanıtılan diğer bir materyal grubu ise zirkonya infiltre lityum silikat (ZLS) seramik bloklardır. Lityum silikatla güçlendirilmiş cam seramikler mekanik açıdan geliştirilerek bu bloklar üretilmiştir. Üretici firmalara göre, materyallerin kırılma direnci 370-420 MPa arasında değişmektedir. ${ }^{29} \mathrm{Bu}$ değerler göz önüne alındığında, ZLS seramiklerin kırılma direncinin, lösit ile güçlendirilmiş cam seramiklerinkinden yüksek olduğu, lityum disilikatla güçlendirilmiş cam seramikler ile $\left(\mathrm{LS}_{2}\right)$ ise kıyaslanabilir düzeyde olduğu söylenebilir. ZLS materyali $\mathrm{LS}_{2}$ seramiklerden; lityum metasilikat ve lityum disilikat kristallerinin daha ince oluşu ve materyalin yapısında zirkonyum oksit içermesi nedeniyle farklıdır. Materyal işlem öncesi prekristalize durumdadır ve sadece lityum metasilikat içerir. Bu aşamada freze işlemleri kolaylıkla yapılabilir. Restorasyon üretildikten sonra $840{ }^{\circ} \mathrm{C}^{\prime} \mathrm{de} 8$ dakika kristalizasyon fırınlaması uygulanır. ${ }^{30}$

Vita Suprinity (Vita Zahnfabrik, Bad Sackingen, Germany); CAD/CAM sistemleri ile kullanılabilen ve içeriğinde; ağırlıkça \%8-12 zirkonya, \%5664 silikon dioksit, \% 15-21 lityum oksit, <\%10 pigmentler bulunan bir malzemedir. İnley, onley, veneer restorasyonlar ve parsiyel kronlar, anterior ya da posterior bölgede tek dişi kapsayan doğal diş üstü ya da implant üstü kronların yapımında kullanılabilmektedir. Yetersiz preperasyon ya da fazla preperasyon sonrası kalan diş dokusunun yetersiz olması durumunda, aşırı çiğneme fonksiyonu ve bruksizmi olan hastalarda özellikle de bu hastaların devital dişlerinde uygulanması önerilmemektedir. Üretici firma tarafından, anterior ve premolar bölgede, köprü restorasyonlarında kullanılmasının teknik açıdan mümkün olabileceği belirtilmiştir. ${ }^{31,32}$

Materyalin yeterli kalınlıkta kullanılmasının fiziksel ve mekanik özellikleri güçlendirdiği bildirilmiştir. Seçilecek veneer materyalinin önemi vurgulanmaktadır. Altyapı ile veneer materyalinin ısısal genleşme katsayısının uyumu sayesinde stres en aza indirilir ve iyi bir bağlantı sağlanabilir. Zirkonya ile güçlendirilmiş 
lityum disilikat cam seramik için üretilen feldspatik düşük ISI porseleni olan 'Vita VM II' tavsiye edilmektedir. ${ }^{32}$

Vita suprinity LS-14 boyutunda T ve HT translüsensi derecelerinde üretilmektedir. İnley, onley, parsiyel kron ve laminate veneerlerde HT bloklar kulanılırken, doğal diş ve implant üstü tam kronlarda $\mathrm{T}$ blokların kullanılmasının daha estetik sonuçlar vereceği bildirilmiştir. ${ }^{33}$ Estetik düzenlemeler ve bitimi; Vita Azkent Plus staining ajanı ile ya da Vita VM 11 materyali ile cut-back tekniğine göre yapılabilir. T bloklarda, staining ve cut-back tekniklerinin her ikisinin de uygulanması uygunken, HT bloklarda staining tekniğinin uygulanması önerilmektedir. Simantasyon aşamasında, restorasyonun, hidroflorik asit ile pürüzlendirilmesi, silan uygulanması ardından rezin siman ile simante edilmesi tesviye edilmektedir. Restorasyonlara $\mathrm{Al}_{2} \mathrm{O}_{3}$ ile kumlama yapılmaması gerektiği belirtilmiştir. $^{34,35}$

Celtra Duo (Dentsply DeTrey, Konstanz, Germany); zirkonya ile güçlendirilmiş lityum silikat seramiktir. Lityum oksit ve silisyum dioksit'e ilave olarak yaklaşık olarak \% 10 zirkonyum dioksit içerir. Zirkonyum oksit, seramik cam faz içerisinde, homojen bir şekilde dağıldığı için, zirkonyum oksit'in kristalizasyonu ve opak görüntüsü engellenmiş olur ve yüksek derecede translüsens bir restorasyon elde edilebilir. ${ }^{35}$ ZLS seramik içindeki kristal partikülleri (0.6-0.8 $\mu \mathrm{m})$, geleneksel lityum disilikat cam seramiklerdeki kristal partiküllerine $(2,5 \mu \mathrm{m})$ kıyasla belirgin ölçüde küçüktür. ${ }^{36}$

\%10'luk zirkonyum içeriği moleküler düzeyde çözünür ve bu materyalin mekanik özelliklerini arttırır, kristalizasyon aşamasında daha kolay aşındırma ve parlatma sağlar, ve restorasyona uygun estetik görünüm sağlayan yüksek saydamlık özelliği kazandırır. Piyasada HT ve LT saydamlık değerlerine sahip bloklar mevcuttur. Simantasyon işlemleri için, sistemin kendine özel asit-bonding ajanı ve translüsens ve medium renk değerlerinde rezin simanı tavsiye edilmekte; ancak diğer adeziv sistemlerin de kullanılabileceği belirtilmektedir. Kişiye özel renk efektleri için Celtra renklendirme ve glazür ürünleri kullanılabilir. Fırınlama işlemleri üreticinin talimatlarına uygun olarak yapılmalıdır. Celtra Duo, glazür fırınlaması esnasında herhangi bir boyutsal değişikliğe uğramamaktadır. ${ }^{37}$

\section{TARTIŞMA}

CAD/CAM sistemleri ile kullanılabilen nanoseramik ve hibrit materyaller ile yapılan çalışmalar incelendiğinde, bu malzemelerin, mekanik özellikler, marjinal uyum, optik parametreler ve biyouyumluluk açısından kabul edilebilir sonuçlar verdiği görülmüştür. CAD/CAM sistemleri ile elde edilen restorasyonların başarısında hekim ve laboratuar çalışanlarının beceri ve tecrübesi kadar kullanılan materyalin mekanik özelliklerini bilmek de önemli rol oynar.

Rezin-seramik CAD/CAM materyallerinin değerlendirildiği bir çalışmada, frezeleme sonrası basamak pürüzlülük değerlerine incelenmiş; rezin-seramik materyallerinin, diğerlerine göre daha üniform basamaklara sahip olduğu ve bu materyallerin, cam seramiklere göre daha konservatif preperasyonlarda kullanılabileceği belirtilmiştir. ${ }^{38}$ Ercan ve ark. tarafından 2015 yılında yapılan bir araştırmada ise; kompozit, nanoseramik ve cam seramik materyallerinden inley restorasyonlar hazırlanmış ve sonuç olarak nanoseramik materyalinin mikrosızıntı değerinin cam seramikler ile benzer özellikte olduğu belirtilmiştir. ${ }^{39}$ Bu bilgiler ışığında nanoseramik ve hibrit materyallerin, uygun endikasyon ile hazırlandığında, mekanik özellikler açısından feldspatik ve $\mathrm{LS}_{2}$ içerikli seramikler ile kıyaslanabilir özelliklere sahip olduğu söylenebilir.

Başka bir çalışmada Lava Ultimate, Cerasmart, Vita Enamic, IPS Empress CAD ve IPS e.max CAD materyallerinin mekanik ve optik özellikleri karşılaştırmalı olarak değerlendirilmiş; Lava Ultimate ve Cerasmart bloklarının bükülme dayanımı, lityum disilikat içerikli seramikten düşük, lösitle güçlendirilmiş cam seramik ve Vita Enamic materyallerinden ise yüksek olarak bulunmuştur. Weibull modülüs değerlendirmelerinde ise kırılganlığı en yüksek materyalin IPS e.max $C A D$, en az olan ise Vita Enamic olduğu tespit edilmiştir. Dolayısıyla, mevcut çalışmaya göre cam seramiklere ait kırılganlık dezavantajı, bu yeni jenerasyon malzemelerde elimine edilmiş gibi görünmektedir. Aynı çalışmada, cam seramik ve Vita Enamic blokları, karşıt dişte, nanoseramik materyallerinden daha fazla aşınma meydana getirmiş ve tüm bu materyaller içinde en az renklenmeye uğrayan materyaller, cam seramikler olmuştur. ${ }^{40}$

Uzun dönem estetik başarının sağlanmasında, renk stabilitesinin korunması önemli rol oynamaktadır. ${ }^{41} \mathrm{Bu}$ konu ile ilgili yapılan bir çalışmada, Lava Ultimate ve Paradigm MZ100 kompozit örnekler, CAD/ 
CAM ile ve geleneksel yöntem ile hazırlanarak, renk stabilitesi yönünden kıyaslanmış; 4 hafta sonunda çeşitli renklendirici solüsyonlarda bekletilen örneklerden CAD/CAM ile elde edilenlerin renklerinin çok daha stabil olduğu bildirilmiştir. Öyleyse, CAD/CAM sistemlerinin kullanılması ile estetik açıdan daha yüksek kalitede restorasyonların elde edilmesi mümkün olabilir. ${ }^{42}$ Estetiğin elde edilmesinde kullanılan teknik kadar malzemenin de önemli olduğu bilinen bir gerçektir. Bu bağlamda farklı malzemelerin (Vita Enamic, Lava Ultimate, IPS e.max CAD, Paradigm $C$ ve Paradigm MZ100) optik özelliklerinin değerlendirildiği başka bir çalışmada; seramik-polimer hibrit materyallerin, polisaj işlemleri sonundaki gloss değerinin, kompozitlerden fazla, saf seramiklerden az olduğu tespit edilmiştir. En az eksternal renklenme seramiklerde görülmüştür. 0 halde estetik gereksinimin yüksek olduğu restorasyonlarda, seramik materyalinin kullanımı ile daha iyi sonuçlara ulaşılabileceği söylenebilir. ${ }^{43}$

Tüm bunların yanı sıra, restorasyonların final rengini; kullanılan seramiğin rengi ve translüsensi değeri etkileyebilmektedir. Estetik CAD/CAM malzemelerinin translüsensi $(T)$ değerlerinin incelendiği bir çalışmada; her bir materyal, farklı kalınlıkta hazırlanmış ve farklı yüzey işlemleri ile pürüzlendirilerek $T$ değerleri ölçülmüştür. En yüksek $T$ değerinin elde edildiği Vita Mark II, diğer materyallerin aksine (Celtra Duo, IPS e.max CAD, IPS Empress CAD, Vita Enamic ve Lava Ultimate), pürüzlendirme sonrası $T$ değerinde değişiklik göstermemiştir. Celtra Duo materyalinde, IPS e.max'ten daha yüksek T değerinde ulaşılmıştır. En düşük $T$ değerine, Vita Enamic malzemesinde ulaşılmıştır. Bunun nedeninin, \%20-23 $\mathrm{Al}_{2} \mathrm{O}_{3}$ içeriği olabileceği belirtilmiştir. Rezin-seramik materyallerin polisaj sonrası $\mathrm{T}$ değeri oldukça iyi iken, pürüzlendirme sonrası, bu değerin, anlamlı derecede düştüğü bildirilmiştir. Öyleyse uzun süreli kullanımda, rezinseramik restorasyonların optik özelliklerinin olumsuz etkileneceği düşünülebilir. ${ }^{44}$

Farklı polisaj tekniklerinin, restorasyon yüzeyi üzerindeki etkilerinin araştırıldığı bir çalışmada; CAD/ CAM tekniği ile onley restorasyonlar hazırlanmıştır. Lava Ultimate ve Vita Enamic restorasyonlara iki farklı teknikle manuel olarak polisaj yapılmış, Empress restorasyonların bir grubuna manuel polisaj, diğer grubuna ise glaze fırınlaması uygulanmıştır. Sonuç olarak Lava Ultimate onleylerde diğer iki materyale göre, daha pürüzsüz yüzeyler elde edilmiştir. Lösitle güçlendirilmiş cam seramik restorasyonlarda her iki teknikle yapılan manuel polisaj sonucunda, glaze fırınlamasına göre daha pürüzsüz yüzeylere ulaşılmıştır. ${ }^{45} \mathrm{Bu}$ çalışmadan elde edilen verilere dayanarak manuel polisaj ile glaze fırınlamasına nazaran daha iyi sonuçlar elde edilebileceği düşünülebilir. Ayrıca seramik-polimer CAD/CAM blokları kullanılarak, geleneksel cam seramikler ile kıyaslanabilir özellikte yüzeylerin, fırınlama gerektirmeksizin daha kısa sürede elde edilebilmesi de önemli bir avantajdır.

\section{SONUÇ}

Seramik-polimer içerikli malzemeler, içeriklerine göre farklılık göstermektedir. Mevcut bilgiler bu materyalleri gruplara ayırmak ve sınıflandırmak için yeterli görünmemektedir. Bu nedenle her bir materyal, kendi mekanik davranışları ve estetik/optik özellikleri doğrultusunda ayrı ayrı incelenmelidir. CAD/CAM sistemlerindeki teknolojik gelişmeler, hekime bağlı başarısızlık nedenlerini azaltmış olsa da tam olarak elimine etmemiştir. Bu sebeple üretilen restorasyonun klinik başarıSı için uyumlama ve bitirme işlemleri titizlikle gerçekleştirilmelidir.

Seramik-polimer materyaller; yeni geliştirilmeleri nedeniyle uzun dönem klinik takipleri henüz mümkün değildir. Daha fazla çalışma yapılarak, bu yeni nesil malzemelerin, başarısını kanıtlamış diğer CAD/ CAM materyalleri ile karşılaştırımaları yararlı olacaktır.

Hamiyet KILINÇ: ORCID ID: 0000-0001-8055-3470

Sedanur TURGUT: ORCID ID: 0000-0003-2534-9279

Elif AYDOĞAN AYAZ: ORCID ID: 0000-0002-9577-5700

Bora BAĞIŞ: ORCID ID: 0000-0003-1974-9719

\section{KAYNAKLAR}

1. Giordano R. Materials for chairside CAD/CAMproduced restorations. J Am Dent Assoc 2006;137:14-21.

2. Beuer F, Schweiger J, Edelhoff D. Digital dentistry: an overview of recent developments for CAD/CAM generated restorations. Br Dent J 2008;204:50511.

3. Karaalioğlu OF, Duymuş ZY. Diş hekimliğinde uygulanan CAD/CAM sistemleri. J Dent Fak Ataturk Uni 2008;18:25-32.

4. Kalaycı BB, Bayındır F. Güncel bilgisayar destekli tasarım/bilgisayar destekli üretim sistemleri. J Dent Fak Ataturk Uni 2015;11:129-36. 
5. Poticny DJ, Klim J. CAD/CAM in-office technology: innovations after 25 years for predictable, esthetic outcomes. J Am Dent Assoc 2010;141:5-9.

6. O'brien W.J. Dental Materials And Their Selection. $3^{\text {th }}$ ed, Quintessence Publishing Co. Inc. Chicago, 2002. P. 48-52.

7. Christensen GJ. In-office CAD/CAM milling of restorations. The future? J Amer Dent Assoc 2008;139:83-5.

8. Li RW, Chow TW, Matinlinna JP. Ceramic dental biomaterials and CAD/CAM technology: State of art. J Prosthodont Res 2014;58:208-16.

9. Liebermann A, Wimmer $T$, Schmidlin PR, Scherer $H$, Löffler $P$, Roos $M$. Physicomechanical characterization of polyetheretherketone and current esthetic dental CAD/CAM polymers after aging in different storage media. J Prosthet Dent 2015;115:321-8.

10. Pop-Ciutrila IS, Dudea D, Badea ME, Moldovan M, Cimpean SI, Ghinea R. Shade Correspondence, Color, and Translucency Differences between Human Dentine and a CAD/CAM Hybrid Ceramic System. J Esthet Restor Dent 2016;28:46-55.

11. Kingery WD, Uhlmann DR, Bowen HK. Introduction to ceramics. $2^{\text {nd }}$ ed. New York: John Wiley \& Sons, 2013. p. 46-76.

12. Saravana KR, Vıjayalakshmı R. Nanotechnology in dentistry. Ind J Dent Res 2006;17:62-5.

13. Kong LX, Peng Z, LI SD, Bartold PM. Nanotechnology and its role in the management of periodontal diseases. Periodontol. 2006,40:184-96.

14. Atabek D, Sillelıoglu $H$, Olmez A. The efficiency of a new polishing material: nanotechnology liquid polish. Oper Dent 2010;35:362-9.

15. Magne P, Paranhos MP, Burnett LH Jr, Magne M, Belser UC. Fatigue resistance and failure mode of novel-design anterior single-tooth implant restorations: influence of material selection for type III veneers bonded to zirconia abutments. Clin Oral Impl Res 2011;22:195-200.

16. Poticny DJ, Klim J. CAD/CAM in-office technology. J Am Dent Assoc 2010;141:5-9.

17. Ab-Ghani Z, Jaafar W, Foo SF, Ariffin Z, Mohamad D. Shear bond strength of computer-aided design and computer-aided manufacturing feldspathic and nano resin ceramics blocks cemented with three different generations of resin cement. J Conserv Dent 2015;18:355-9.
18. Koizumi H, Saiki O, Nogawa H, Hiraba H , Okazaki $T$, Matsumura $H$. Surface roughness and gloss of current CAD/CAM resin composites before and after toothbrush abrasion. Dent Mater J 2015;34:881-7.

19. Lava $^{\text {TM }}$ Ultimate CAD/CAM Restorative Instructions for Use, 3M ESPE Dental Products, St. Paul United States. 2016;www.3m.com.

20. GC Cerasmart Product Description. 2014; 800.323.7063 www.gcamerica.com.

21. Güth JF, Zuch T, Zwinge S, Engels J, Stimmelmayr $M$, Edelhoff D. Optical properties of manually and CAD/CAM-fabricated polymers. Dent Mater J 2013;32:865-71.

22. Magne P, Paranhos MP, Burnett LH Jr, Magne M, Belser UC. Fatigue resistance and failure mode of novel-design anterior single-tooth implant restorations: influence of material selection for type III veneers bonded to zirconia abutments. Clin Oral Impl Res 2011;22:195-200.

23. Acar Ö. Farklı yüzey hazırlıklarının CAD/CAM hibrit seramiğin kompozit rezin ile tamirine etkisi. Acta Odontologica Turcica 2016;33:121-5.

24. Chen C, Trindade FZ, de Jager N, Kleverlaan CJ, Feilzer AJ. The fracture resistance of a CAD/CAM resin nano ceramic and a CAD ceramic at different thicknesses. Dent Mater 2014;30:954-62.

25. Johnson AC, Versluis A, Tantbirojn D, Ahuja S. Fracture strenght of CAD/ CAM composite and composite-ceramic occlusal veneers. J Prosthodont Res 2014;58:107-14.

26. Dupriez ND, Koeckritz AK, Kunzelmann KH. A comparative study of sliding wear of nonmetallic dental restorative materials with emphasis on micromechanical wear mechanisms. J Biomed Mater Res B Appl Biomater 2015;103:925-34.

27. Coldea A, Swain MV, Thiel N. Mechanical properties of polymer-infiltrated-ceramic-network materials. Dent Mater 2013;29:419-26.

28. Dirxen C, Blunck U, Preissner S. Clinical performance of a new biomimetic double network material. Open Dent J 2013;7:118-22.

29. Della Bona A, Corazza PH, Zhang Y. Characterization of a polymer-infiltrated ceramicnetwork material. Dent Mater 2014;30:564-9. 
30. Güth JF, Zuch T, Zwinge S, Engels J, Stimmelmayr M, Edelhoff D. Optical properties of manually and CAD/CAM-fabricated polymers. Dent Mater J 2013;32:865-71.

31. Pieger S, Salman A, Bidra AS. Clinical outcomes of lithium disilicate single crowns and partial fixed dental prostheses: a systematic review. J Prosthet Dent 2014;112:22-30.

32. Working Instructions of VITA Suprinity. VITA Zahnfabrik H. Rauter GmbH \& Co. KG Postfach 1338, Bad Säckingen, Germany, 2014; D-54812 www.vita-zahnfabrik.com.

33. Awad D, Stawarczyk B, Liebermann A, Ilie N.Translucency of esthetic dental restorative CAD/CAM materials and composite resins with respect to thickness and surface roughness. J Prosthet Dent 2015;113:534-40.

34. Frankenberger $R$, Hartmann VE, Krech $M$, Krämer $N$, Reich S, Braun A, Roggendorf $M$. Adhesive luting of new CAD/CAM materials. Int J Comput Dent 2015;18:9-20.

35. D'Arcangelo C, Vanini L, Rondoni GD, De Angelis F. Wear properties of dental ceramics and porcelains compared with human enamel. J Prosthet Dent 2016;115:350-5.

36. Frankenberger $\quad R$, Zeilinger $I$, Krech $M$, Mörig G, Naumann M, Braun A, Krämer N, Roggendorf MJ. Stability of endodontically treated teeth with differently invasive restorations: Adhesive vs. nonadhesive cusp stabilization. Dent Mater 2015; 31:1312-20.

37. Preis V, Behr M, Hahnel S, Rosentritt M. Influence of cementation on in vitro performance, marginal adaptation and fracture resistance of CAD/CAMfabricated ZLS molar crowns. Dent Mater 2015; 31:1363-9.

38. Awada A, Nathanson D. Mechanical properties of resin-ceramic CAD/CAM restorative materials. ] Prosthet Dent 2015;114:587-93.

39. Ercan E, İbrahimova D, Hamidi MM. Kompozit inley, indirekt porselen inley ve cad/cam inley sistemi ile restore edilen dişlerin kenar sızıntılarının incelenmesi: in vitro çalışma. 2004;14:59-66.

40. Alberto A, Pascual A, Camps I, Grau-Benitez M. Comparative characterization of a novel cad-cam polymer-infiltrated-ceramic-network. J Clin Exp Dent 2015;7:495-500.
41. Battersby PD, Battersby SJ. Measurements and modelling of the influence of dentine colour and enamel on tooth colour. 2015;43:373-81.

42. Arocha M, Basilio J, Llopis J. Colour stainability of indirect CAD/CAM processed composites vs conventionally laboratory processed composites after immersion in staining solutions. J Prosthet Dent 2014;8:831-8.

43. Lawson NC, Burgess JO.Gloss and Stain Resistance of Ceramic-Polymer CAD/CAM Restorative Blocks. J Esthet Restor Dent. 2016;28:40-5.

44. Awad A, Stawarczyk B, Liebermann A, Eichberger $M$, Güth JF. Evaluation of mechanical and optical behavior of current esthetic dental restorative CAD/CAM composites. J Mech Behav Biomed Mater 2015; 55:1-11.

45. Fasbinder DJ, Neiva GF. Surface Evaluation of Polishing Techniques for New Resilient CAD/CAM Restorative Materials. J Esthet Restor Dent 2016;28:56-66.

\section{Yazışma Adresi}

Dr. Öğr. Üyesi Hamiyet KILINÇ

Bolu Abant İzzet Baysal Üniversitesi,

Diş Hekimliği Fakültesi,

Protetik Diş Tedavisi AD, Bolu

05067716621

e-mail: hamiyetonder@windowslive.com

hamiyetkilinc@ibu.edu.tr 